\title{
Nonperturbative treatment of the screened-Coulomb contribution of projectile-electron loss
}

\author{
P. L. Grande,$^{1}$ G. Schiwietz, ${ }^{2}$ G. M. Sigaud, ${ }^{3}$ and E. C. Montenegro ${ }^{3}$ \\ ${ }^{1}$ Instituto de Física da Universidade Federal do Rio Grande do Sul, Avenida Bento Goncalves 9500, 91501-970, Porto Alegre, Brazil \\ ${ }^{2}$ Bereich F, Hahn-Meitner-Institut Berlin, Glienicker Strasse 100, D-14109 Berlin, Germany \\ ${ }^{3}$ Departamento de Física, Pontificia Universidade Católica do Rio de Janeiro, Caixa 38071, \\ Rio de Janeiro, Rio de Janeiro 22453-970, Brazil
}

(Received 1 May 1996)

\begin{abstract}
The electron-loss cross section of $\mathrm{He}^{+}$ions impinging upon noble-gas targets $\left(2 \leqslant Z_{2} \leqslant 36\right)$ is calculated by using the coupled-channel method for the active projectile electron in the static screened field of the target atom. The calculations show a saturation of the projectile-electron-loss cross section with increasing target atomic number. This saturation effect due to neutral target systems is much more pronounced than for ionization or excitation by charged particles. Comparison with experimental data indicates a small electron-loss contribution from electron-electron interaction processes for heavy targets at intermediate velocities. Remaining discrepancies in the data are discussed in the light of the approximations involved in our theoretical treatment. [S1050-2947(96)11009-X]

PACS number(s): $34.50 . \mathrm{Fa}$
\end{abstract}

\section{INTRODUCTION}

The electron-loss process of projectile ions interacting with neutral atoms is determined by two competing mechanisms that depend on the role played by the target electrons during the collision. They can either weaken the electric field produced by the target nucleus or directly ionize the projectile through the electron-electron interaction. These two contributions are usually denoted by screening and antiscreening effects $[1,2]$. The projectile electron-loss arising from the interaction with the target nucleus screened by the target electrons, the so-called screening or screened-Coulomb contribution, predominates at low velocities and small impact parameters. At high incident energies simultaneous projectile ionization and target excitation and/or ionization through the electron-electron interaction strongly enhances the projectile electron loss, a process which has been called doubly inelastic or dielectronic transition or antiscreening effect [1-3]. The first description of such electron-loss processes was due to Bates and Griffing [4] within the first-order Born approximation.

Recent investigations have shown that the behavior of the screened-Coulomb and the electron-electron contributions can be satisfactorily understood in the intermediate-to-high velocity regime within the plane-wave Born approximation (PWBA) framework for light targets [2,5]. This does not hold true for heavier targets. When the target atomic number, $Z_{2}$, increases, first-order theories predict that the loss cross section for the screened-Coulomb contribution should increase approximately as $Z_{2}^{2}$. In contrast, the experimental projectile electron-loss cross section shows a pronounced saturation for target elements heavier than $\mathrm{Ne}$ [6]. This comes from the fact that the ionization probability cannot increase indefinitely as a function of $Z_{2}$, since it cannot exceed 1. Each electronic transition gives rise to an increased final-state population and a corresponding reduction of the initial-state population. This reduction is not accounted for in first-order theories and leads to the artificial creation of elec- trons (overestimated cross section). In the case of target ionization, the cross sections are determined by ionization probabilities from significantly larger impact parameters. Large impact parameters correspond to small ionization probabilities and correspondingly there are no strong deviations from the predictions of first-order perturbation theory. Hence, especially for electron loss by neutral atoms (small impact parameters), more elaborate theories such as nonperturbative calculations have to be applied.

In this work we use the coupled-channel method $[7,8]$ to calculate the screened-Coulomb contribution to the electronloss cross section for $\mathrm{He}^{+}$ions incident on $\mathrm{He}, \mathrm{Ne}, \mathrm{Ar}$, and $\mathrm{Kr}$ targets, in the energy range from 250 to $1000 \mathrm{keV} / \mathrm{u}$ (corresponding to velocities ranging from 3.16 to 6.32 a.u.). We have chosen these systems and energies since they are the same as those measured by Sant'Anna et al. [6] and comprise the energy threshold for the electron-electron contribution. If not indicated otherwise, atomic units (a.u.) will be used throughout the paper.

\section{THEORY}

In a full quantum-mechanical description, the ion-atom collision process is described by the many-body Schrödinger equation. For incident energies above a few $\mathrm{eV} / \mathrm{u}$ the motion of the nuclei may be described by classical trajectories, characterized by the internuclear distance $\vec{R}(t)$ [9]. Under this assumption, the electronic system obeys the time-dependent Schrödinger equation, [9]

$$
\mathcal{H}_{e}(\vec{R}(t)) \Phi_{e}(\{\vec{r}\}, t)=i \frac{\partial}{\partial t} \Phi_{e}(\{\vec{r}\}, t)
$$

where $\{\vec{r}\}$ represents the set of electronic coordinates. In this work, the nuclear trajectories $\vec{R}(t)$ are assumed to be straight lines, $\vec{R}=\vec{v} t+\vec{b}$, determined by the relative velocity $\vec{v}$ and the impact parameter $\vec{b}$. Unless otherwise indicated, we are 


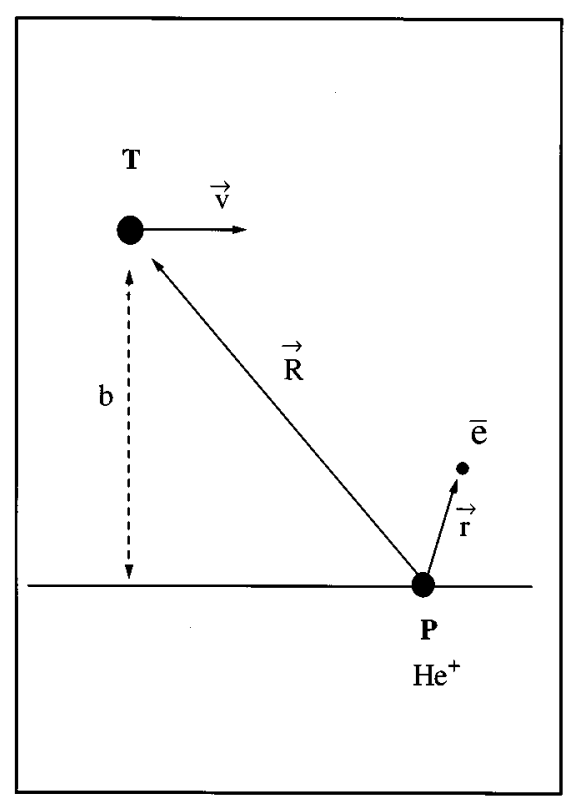

FIG. 1. Sketch of the collision process in the projectile frame. The active-electron coordinate is represented by $\vec{r}$. The target atom following a straight-line trajectory specified by $\vec{R}(t)$ provides a time-dependent perturbation on the projectile electron. The targetatom trajectory is determined by the impact parameter $b$ and relative velocity $v$.

considering the reference system where the projectile is at rest (projectile-frame) (see Fig. 1).

The electronic many-body Hamiltonian in Eq. (1) is treated in the framework of the independent-electron approximation. This means that there is only one active electron. Therefore, we have to adopt different approaches in order to take into account all mechanisms leading to the projectile-electron loss. As mentioned above, we divide the electron-loss contributions into two parts depending on the role played by the target electrons.

In the case in which the target electrons just screen the target nucleus (screened-Coulomb contribution) we solve the time-dependent Schrödinger equation (1) through the coupled-channel method assuming that the target electrons remain in the ground state. Thus, the target electrons just provide a time-dependent perturbation on the active projectile electron through their static mean field. Since this mechanism does not involve an explicit correlation between the electrons in the two centers (projectile ion and target atom), the screened-Coulomb contribution will be responsible for uncorrelated transitions. In what follows, the probability of this contribution as a function of the impact parameter will be called $P_{\text {uncorr }}(b)$. Changes in the screened target potential due to the interaction with the projectile (dynamic screening) will be neglected. At this point it should be noted that doubly or multiply inelastic transitions are also possible as a result of uncorrelated interactions in higher orders of the perturbation (even in an independent-electron description). Thus, we generally avoid the use of this term in connection with correlated electron-electron processes.

The electron-electron interaction between the bound projectile electrons and the target electrons is taken into account here in first-order perturbation theory, since each electronelectron interaction represents only a small perturbation for the impact velocities studied in this work. The corresponding effect results in an enhancement of the ionization and excitation cross sections at intermediate-to-high energies $[1,2,4]$. Here, the transitions due to electron-electron interaction are taken into account by using the procedure of Montenegro and Meyerhof [10], which is based on the plane-wave Born treatment and on an improved closure approximation to sum over all target final states. The corresponding electronic transitions will be named correlated transitions in this work, since they result in a simultaneous transition of the interacting electrons in the two colliding systems. The impactparameter dependence of the probability of such transitions $P_{\text {corr }}(b)$ is calculated according to the method in Ref. [2] using an effective ion velocity independent of the final projectile-electron state. This method gives the same electron-loss cross section as calculated from the procedure of Montenegro and Meyerhof [10] within the PWBA.

In what follows, we give a brief description of the coupled-channel method used to calculate the probabilities for uncorrelated transitions (screened-Coulomb contribution) and the total probabilities for electron loss.

\section{A. Coupled-channel calculations}

The time-dependent Schrödinger equation (1) is solved by expanding $\Phi_{e}(\{\vec{r}\}, t)$ in terms of unperturbed eigenfunctions $\phi_{i}$ of the projectile with coefficients $a_{i}(t)=\left\langle\phi_{i} \mid \Phi_{e}(t)\right\rangle$. Thus, Eq. (1) is replaced by a set of coupled first-order differential equations, the so-called coupled-channel equations:

$$
i \frac{d}{d t} a_{i}(t)=\sum_{j} a_{j}(t) e^{i \omega_{i, j} t} V_{i n t}^{i, j}(\vec{R}(t)), \quad \omega_{i, j}=E_{i}-E_{j}
$$

with

$$
V_{i n t}^{i, j}(\{\vec{R}(t)\})=\left\langle\phi_{i}\left|V_{i n t}(\vec{R}(t)-\vec{r})\right| \phi_{j}\right\rangle .
$$

$E_{i}$ is the orbital energy associated with the projectile wave function $\phi_{i}$. Here $V_{i n t}$ is an effective potential seen by the active electron, which contains the Coulomb part due to the target-nuclear charge and the static potential produced by the target electrons that screen the target-nuclear charge,

$$
V_{i n t}(\vec{R}-\vec{r})=-\frac{Z_{2}}{|\vec{R}-\vec{r}|}+\sum_{n}^{\mathcal{N}} \int d^{3} r^{\prime} \frac{\left|\Phi_{n}\left(\overrightarrow{r^{\prime}}\right)\right|^{2}}{\left|\vec{R}-\vec{r}-\overrightarrow{r^{\prime}}\right|},
$$

where $Z_{2}$ is the target nuclear charge, $\Phi_{n}$ is the targetelectron wave function and $\mathcal{N}$ is the number of target electrons. The wave functions $\Phi_{n}$ for each electron $n$ of the target are obtained according to the Hartree-Fock-Slater procedure [11]. Thus, we neglect dynamic screening (a time dependence of $\Phi_{n}$ due to target polarization, respectively, excitation and/or ionization), Pauli correlation (antisymmetrization of the projectile- and target-centered wave functions), and dynamic correlation effects due to the residual electron-electron interaction.

In order to integrate the coupled-channel equations (2) the time as well as the impact-parameter dependence of the matrix elements (3) have to be determined. For this purpose, the 
matrix elements $V_{i n t}^{i, j}(\{\vec{R}(t)\})$ are expanded in terms of the radial $(R)$ and angular $(\hat{R})$ parts of the internuclear vector $\vec{R}$ according to

$V_{i n t}^{i, j}(\vec{R})=-\sum_{L=\left|l_{i}-l_{j}\right|}^{l_{i}+l_{j}} W_{L, M}^{i, j} G_{L}^{i, j}(R) Y_{L, M}(\hat{R}), \quad M=m_{j}-m_{i}$,

which is obtained after separating the radial and angular parts (determined by the spherical harmonics $Y_{L, M}$ ) of the atomic projectile wave function $\phi_{i}$. The coefficients $W_{L, M}^{i, j}$ are given by

$$
\begin{aligned}
W_{L, M}^{i, j}= & \left(\frac{4 \pi\left(2 l_{i}+1\right)\left(2 l_{j}+1\right)}{2 L+1}\right)^{1 / 2}(-1)^{m_{i}+M} \\
& \times\left(\begin{array}{ccc}
l_{i} & l_{j} & L \\
0 & 0 & 0
\end{array}\right)\left(\begin{array}{ccc}
l_{i} & l_{j} & L \\
-m_{i} & m_{j} & -M
\end{array}\right) .
\end{aligned}
$$

In the above equation $l$ and $m$ are the quantum numbers associated with angular momentum and angular momentum projection, respectively. The symbols $(\ldots \ldots \ldots \ldots)$ in Eq. (6) represent the Wigner ' $3 j$ ', symbol as described in Ref. [12]. We consider only screened interaction potentials which are spherically symmetric. In this case the function $G_{L}^{i, j}(R)$ can be written as

$$
G_{L}^{i, j}(R)=\int_{0}^{\infty} d r r^{2} \chi_{i}^{*} \chi_{j} f_{L}(r, R)
$$

where $\chi_{i}, \chi_{j}$ are the radial wave functions of the states $i$ and $j$, respectively. The function $f_{L}(r, R)$ is determined by the interaction potential only and reads for the Bohr-like screened potential $\exp (-\lambda|\vec{R}-\vec{r}|) /(|\vec{R}-\vec{r}|)$,

$$
f_{L}(r, R)=\lambda(2 L+1)(-1)^{L} A_{L}\left(\lambda r_{<}\right) H_{L}\left(\lambda r_{>}\right),
$$

where $A_{L}(x) \equiv i^{L} j_{L}(i x)$ and $H_{L}(x) \equiv i^{L+1} h_{L}^{+}(i x)$ with $j_{L}(i x)$ and $h_{L}^{+}(i x)$ being the modified spherical Bessel functions [13]. The notation $r_{<(>)}$means the smaller (larger) of the values of $r$ and $R$. In the case of an unscreened interaction $f_{L}(r, R)$ is given by $r_{<}^{L} / r_{>}^{L+1}$, according to the wellknown multipole expansion of the Coulomb potential.

Here, the screened potential (4) for neutral target atoms is represented by

$$
V_{\text {int }}(\vec{R}-\vec{r})=\sum_{n=1}^{n_{\max }}\left(A_{n}+B_{n}|\vec{R}-\vec{r}|\right) \frac{\exp \left(-\lambda_{n}|\vec{R}-\vec{r}|\right)}{|\vec{R}-\vec{r}|},
$$

where the coefficients $A_{n}, B_{n}$, and $\lambda_{n}$ are obtained by fitting to the numerically determined potential from Eq. (4). The number of Bohr-like screened potential terms, $n_{\max }$, corresponds to the number of electronic shells of each target atom. In this way, the function $f_{L}(r, R)$ used in Eq. (7) is obtained straightforwardly.

With the matrix elements from Eq. (5) the coupledchannel equations are solved numerically in order to obtain the coefficients $a_{i}$ after the collision $(t \rightarrow \infty)$. The probability of ionizing the projectile from the ground state to a continuum state of energy $\epsilon$, angular momentum $l$, and projection $m$ in a collision with impact parameter $\mathrm{b}$ is given by

$$
\frac{d P_{l, m}}{d \epsilon}(b)=\lim _{t \rightarrow \infty}\left|a_{\epsilon, l, m}(b, t)\right|^{2}
$$

and the ionization probability is calculated according to

$$
P_{\text {uncorr }}(b)=\sum_{l, m} \int_{0}^{\infty} d \epsilon \frac{d P_{l, m}}{d \epsilon}(b),
$$

which corresponds to the loss probability due to uncorrelated transitions.

In the atomic-orbital (AO) expansion used, unlike the perturbative approaches, the sum over the population probabilities of all states is always 1 . In the latter approaches even for $\mathrm{H}^{+}+\mathrm{H}$ collisions (e.g., $20 \mathrm{keV}$ ) the total ionization probability may exceed 1 at small impact parameters. Thus, a saturation of ionization probabilities is expected in our calculations for increasing perturbing field.

We can also restrict the computer code used to the socalled SCA, semiclassical approximation [14]. In this approach, the coupled-channel equations are solved by neglecting all matrix elements, except those which lead to transition from the initial state ( $1 s$ for He projectiles) to one of the final states. At projectile energies far above the ionization potentials involved, the corresponding results are identical to those from the first-order plane-wave Born approximation.

As discussed above, the advantages of coupled-channel calculations compared to first-order theories (SCA, PWBA) should appear more clearly at intermediate velocities and small impact parameters. In contrast to most other coupledchannel calculations [15] we have used a large number of bound states as well as continuum wave packets composed of a superposition of continuum eigenstates (up to 500 gerade states with partial waves up to $l=8$ ), since the correct computation of ionization probabilities for screened interaction potentials demands high accuracy of the emitted electron energy-spectrum.

Further details of the calculation, e.g. the numerical treatment of continuum states and adopted basis set, may be found in Refs. [7,8].

\section{B. Total probabilities for the electron loss}

In order to obtain the total electron-loss probabilities we must consider the uncorrelated and correlated contributions. As noted in the last section, the use of the coupled-channel method to calculate the uncorrelated probability, $\mathrm{P}_{\text {uncorr }}$ implies that the sum over all states is necessarily 1 (unitarity). Thus, the proper inclusion of the contribution due to correlated processes must be performed in such a way as to keep this unitarity to the total electron-loss probability. Of course this implies that the probabilities due to both these contributions cannot be simply added.

The uncorrelated probability, calculated through the AO expansion, represents the probability of removal of the active projectile active electron due to the projectile-electrontarget-nucleus interaction. In this way, $\left(1-P_{\text {uncorr }}\right)$ corresponds to the probability of nothing happening to the projec- 
tile electron in spite of its interaction with the screened target nucleus. If we assume that the uncorrelated probability is not altered in the presence of the electron-electron processes ( in other words, statistical independence of both processes), then the electron-electron contribution must come from its complement, i.e., $\left(1-P_{\text {uncorr }}\right)$. In the case of two active electrons, one on each collision partner, the total electronloss probability, $P_{\text {loss }}$ is

$$
\begin{aligned}
P_{\text {loss }} & =P_{\text {uncorr }}+\left(1-P_{\text {uncorr }}\right) P_{\text {corr }}, \\
& =1-\left(1-P_{\text {uncorr }}\right)\left(1-P_{\text {corr }}\right) .
\end{aligned}
$$

This procedure can be generalized to the case where the target has more than one electron. For the total electron-loss cross section we can then write

$$
\begin{aligned}
\sigma_{\text {tot }}= & \int_{0}^{\infty} 2 \pi b d b\left(1-\left[1-P_{\text {uncorr }}(b)\right] * \prod_{i}\right. \\
& \left.\times\left[1-P_{\text {corr }}^{i}(b)\right]^{n_{i}}\right),
\end{aligned}
$$

where $P_{c o r r}^{i}(b)$ is the probability per electron for correlated transitions due to $n_{i}$ electrons from the $i$ th shell of the target atom.

\section{RESULTS AND DISCUSSION}

In Figs. 2 to 5 we present the comparison between the calculated total electron-loss cross section [Eq. (14)], upper solid line) and the experimental data of Ref. [6] as a function of the projectile energy. The theoretical curves for the screened-Coulomb contribution are our atomic-orbital coupled-channel calculations according to Eq. (2) (dashed line) and the first-order Born approximation [16] (dotted line). The contribution due to correlated electron-electron interactions (dash-dotted line) was calculated according to the procedure of Montenegro and Meyerhof [10]. All calculations were performed using Hartree-Fock-Slater [11] wave functions for the description of the target electrons.

It can be noticed that the results of Montenegro and Meyerhof follow roughly the trends of the experimental data as a function of the energy for all targets. This behavior has also been observed for heavier projectiles like $\mathrm{C}^{3+}$ [17] at lower projectile velocities.

As expected, the coupled-channel and PWBA calculations for uncorrelated processes give approximately the same results for the He target, except for the lowest energies. However, the difference between both results increases with increasing target atomic number (compare Figs. 2-5), reaching almost one order of magnitude for the $\mathrm{Kr}$ target. This is a consequence of a saturation of the screened-Coulomb contribution, which indicates the breakdown of first-order perturbation theory. It is seen that the coupled-channel results for uncorrelated transitions agree with the experimental total cross sections for $\mathrm{Ne}$ and Ar to within the experimental uncertainty. For the $\mathrm{Kr}$ target, the coupled-channel results exceed the experimental data by about $30 \%$ at low incident energies.

The total cross sections agree very well with the experimental data for the He target in the whole energy range.

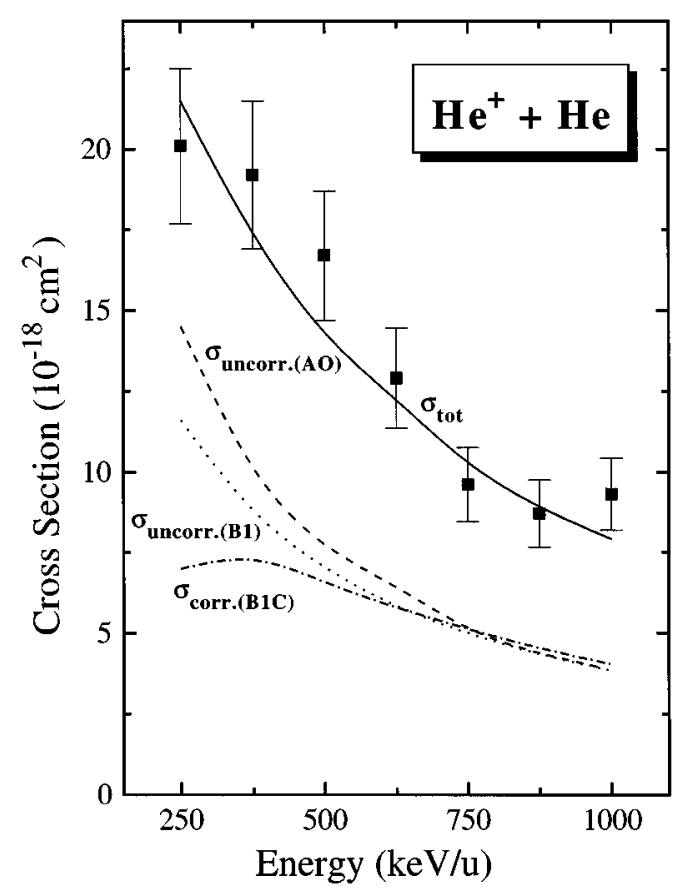

FIG. 2. $\mathrm{He}^{+}$electron-loss cross section as a function of the scaled ion energy for $\mathrm{He}, \mathrm{Ne}, \mathrm{Ar}$, and $\mathrm{Kr}$. The full squares correspond to the total cross section measurements of Sant'Anna et al. from Ref. [6]. The dashed line (AO) represents the coupled-channel results for the uncorrelated electron loss cross section. For comparison a PWBA calculation for the uncorrelated contribution (firstorder-Born electron-loss cross section $\left.\sigma_{u n c o r r}(B 1)\right)$ is shown by a dotted line. The dash-dotted line corresponds to the calculation of the correlated electron-electron processes using PWBA plus closure approximation (first-order-Born-Closure electron-loss cross section $\left.\sigma_{\operatorname{corr}(B 1 C)}\right)[10]$. The estimated total electron cross section $\left(\sigma_{t o t}\right)$ is calculated according to Eq. (14).

Since the probabilities for correlated and uncorrelated transitions are small, there is no significant difference between the electron-loss cross section given by Eq. (14) and the simple sum of the cross sections of correlated and uncorrelated processes. Nevertheless, for other target atoms the simple sum of cross sections (this cross section is not shown in the figures) is 7-12\% larger than the total cross section given by Eq. (14) (upper solid lines) depending on the ion velocity and target atom. For the Ne target (see Fig. 3) the agreement between the present theoretical calculations and experimental cross sections is reasonable with a systematic deviation of about $15-20 \%$. As $Z_{2}$ increases, the present calculations for the total electron-loss cross section overestimates the experimental results significantly (by up to about $60 \%$ for $\mathrm{Kr}$ at low energies). This may be due to different reasons.

First, the calculated screening contribution presents a difficulty, that is intrinsic to the independent particle model (IPM) that we have adopted for the coupled-channel calculations. Since we use a large number of projectile-centered basis states to accurately describe the electron loss, this basis can include combinations that simulate unrealistic electron capture into occupied bound states of the target (this is in contradiction to the Pauli exclusion principle). In Fig. 6 we present the time-dependent projectile-electron density pro- 


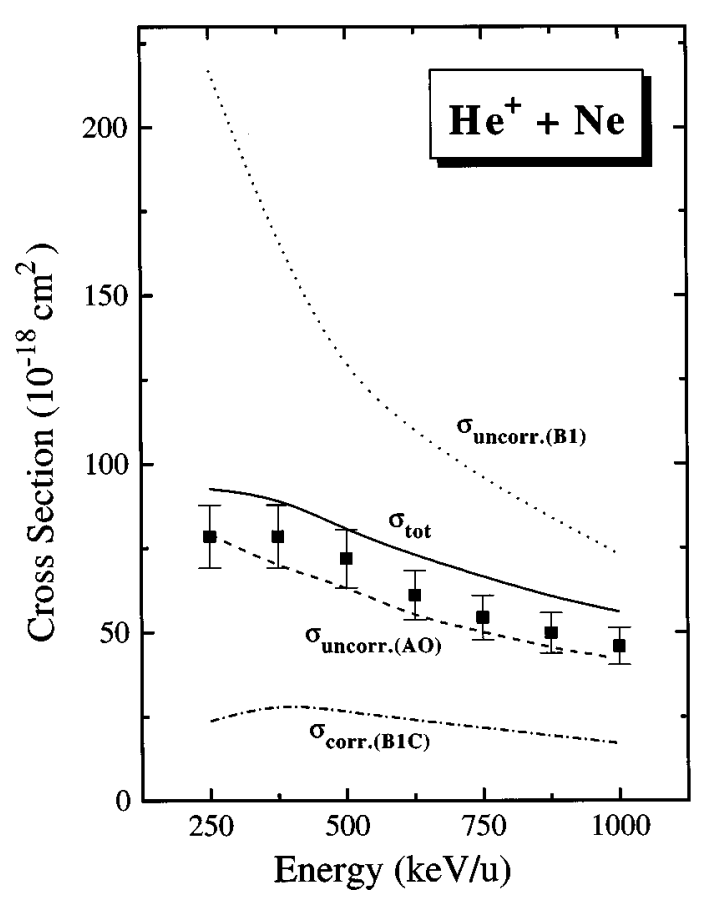

FIG. 3. Same as in Fig. 2.

jected on the collision plane for an impact parameter of 2 a.u. only shortly after the $\mathrm{Kr}$ target, represented by a full circle, has passed the distance of closest approach. The position corresponds to an internuclear distance $R$ of about 3 a.u. The time-dependent electronic density was obtained directly from the calculated transition amplitudes $a_{i}(t)$ according to

$$
\rho(\vec{r}, t)=\sum_{i, j} a_{i} a_{j}^{*} e^{-i\left(E_{i}-E_{j}\right) t} \phi_{i}(\vec{r}) \phi_{j}^{*}(\vec{r}) .
$$

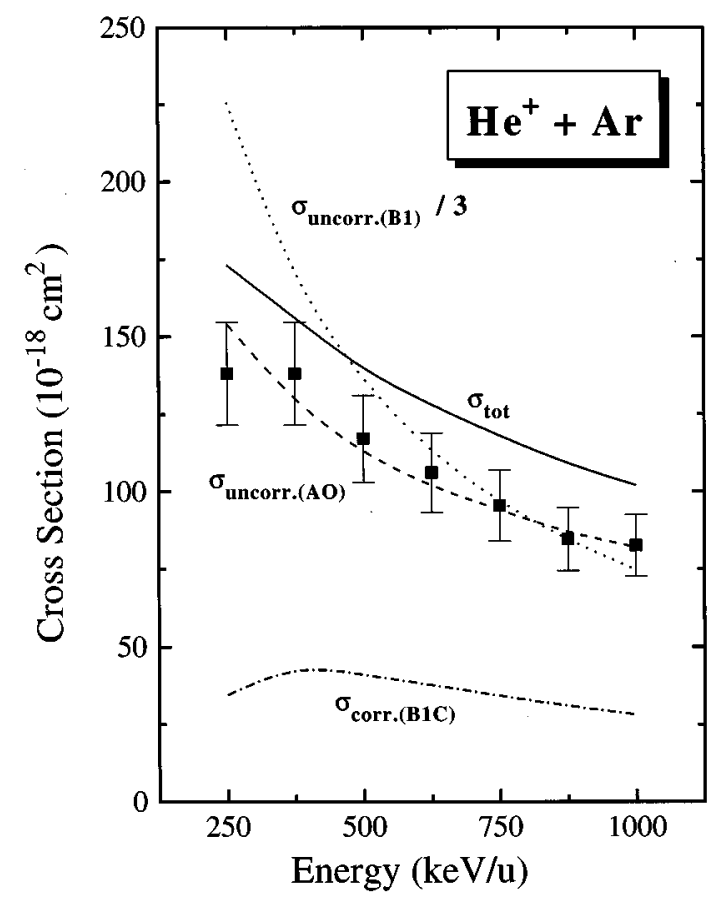

FIG. 4. Same as in Fig. 2.

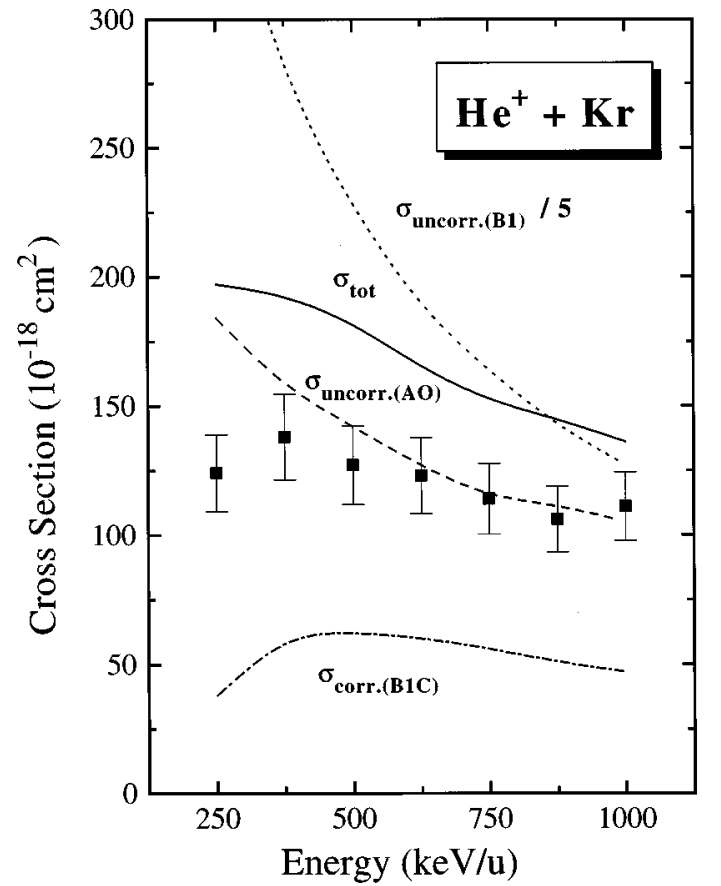

FIG. 5. Same as in Fig. 2.

The electron cloud is attracted by the traveling target nucleus up to a distance of about 5 a.u. For larger distances the adopted basis set is not complete enough to describe a target-centered state (corresponding to electron capture). Another signature of this spurious capture process emerges in the singly differential cross section (see Fig. 7), where a peak around $v^{2} / 2$ ( $v$ is the target velocity in the projectile frame) is found. This effect turns out to be much stronger for the $\mathrm{Kr}$

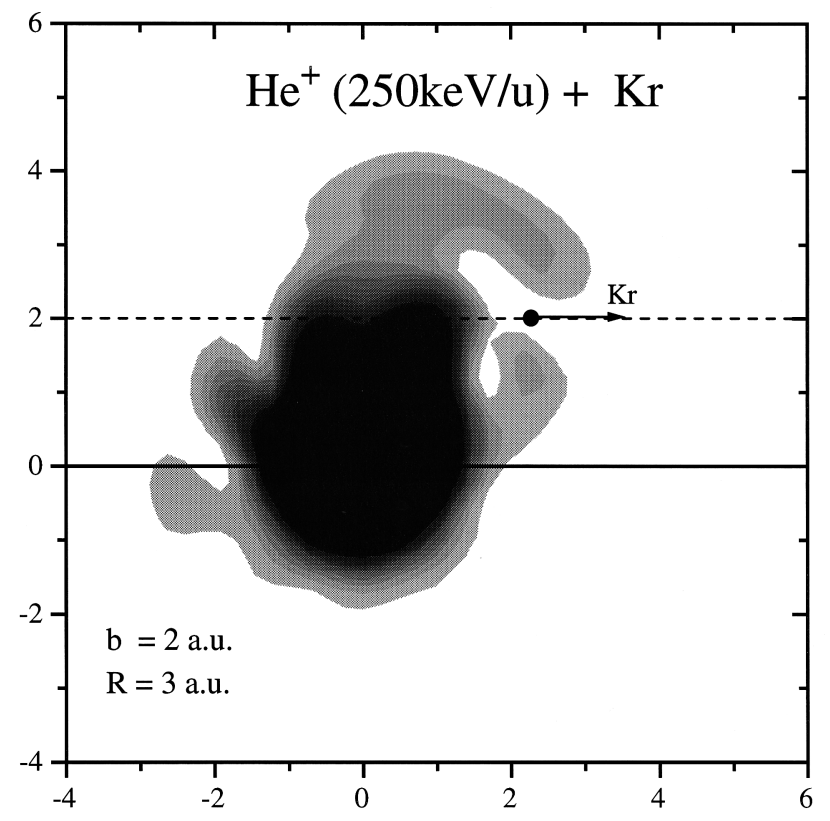

FIG. 6. Projection of the time-dependent projectile-electron density on the collision plane for a collision of $\mathrm{He}^{+}$with a $\mathrm{Kr}$ atom (full circle) at a proton-equivalent energy of $250 \mathrm{keV} / \mathrm{u}$ and for an impact parameter of 2 a.u. 


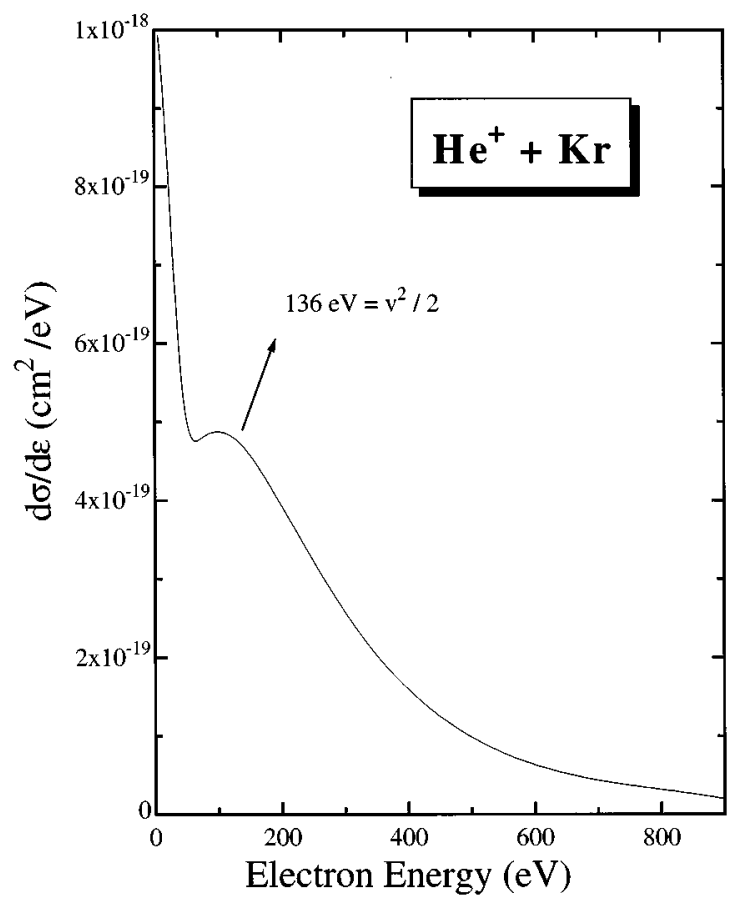

FIG. 7. Coupled channel results for the singly differential cross section of $\mathrm{He}^{+}$due to the impact of $\mathrm{Kr}$ atoms at the energy of 250 $\mathrm{keV} / \mathrm{u}$.

target than for the $\mathrm{He}, \mathrm{Ne}$, and Ar targets, since the binding energy for an electron in the screened $\mathrm{Kr}$ field corresponding to the $3 d$ shell $(44.5 \mathrm{eV})$ is close to the $1 s \mathrm{He}^{+}$binding energy $(54.4 \mathrm{eV})$. Moreover, this unrealistic capture process is important only for lower energies, where the capture cross section is high, as can be readily seen from Fig. 5. Although this unrealistic capture decreases with increasing energy, it may give a significant contribution for $\mathrm{Kr}$ at higher energies, too. However, this point of criticism applies to $\mathrm{Kr}$ targets only because of the near-resonant capture condition and the large target-nuclear charge $Z_{2}$.

The second reason for the discrepancy between experiment and theory concerns the calculation of the probabilities for correlated transitions. It is well known that the closure procedure of Montenegro and Meyerhof [10] overestimates the electron-electron contribution for intermediate projectile energies [10] (e.g., by $25 \%$ for $100-\mathrm{keV} \mathrm{H}^{0}+\mathrm{H}$ ). For $\mathrm{He}^{+}$ on light targets we expect uncertainties on the order of only $10 \%$ for the electron-loss contribution, since the closure approximation should be more accurate for higher projectile binding energies. However, for many-electron targets it is not clear how accurate the antiscreening cross section is predicted. Increased electronic binding as well as polarization due to the presence of both nuclei may also be important.

One additional approximation, not discussed so far, is the neglect of target polarization for the correlated as well as for the uncorrelated loss processes. Target electrons with high orbital velocity, e.g., $\mathrm{Ne}-K$, Ar- $K$, and at lower projectile velocities also $\operatorname{Ar}-L$ shell electrons, may adjust adiabatically to the projectile motion. For the case of uncorrelated transitions this dynamic screening involves a nonspherical target potential and a corresponding reduction of the effective target charge. For $\mathrm{He}^{+}+\mathrm{Ne}$ collisions we estimate a lowering of the uncorrelated cross sections on the order of $2 \%$ due to this effect. For the Ar target this reduction may be about $5 \%$. The correlated contribution is expected to be slightly enhanced due to target polarization.

Another reason for the discrepancy is related to the electron-loss measurements. The electron loss may be accompanied by electron capture (capture of a target electron by the projectile) in the same collision event, resulting in a final charge state equal to the initial one. Such a process is experimentally not identified since only the final charge state of the projectile was measured [6]. In fact, this process can hardly be separated from electron capture into projectilecentered continuum states. Hence, the exclusion of electron capture associated with electron loss has to be performed within the theoretical treatment. Following the procedure of Ref. [2], the probability for uncorrelated transitions $P_{\text {uncorr }}(b)$ should be multiplied by the probability of not capturing any target electrons. In the same way, the probability for correlated transitions $P_{c o r r}(b)$ should be multiplied by the probability of not capturing the other target electrons that are not participating in the electron-electron process. Estimates using the boundary-corrected first-order Born [18] and Oppenheimer-Brinkman-Kramers [19] impact-parameter dependence of the capture probability, normalized to experimental cross sections of Ref. [20], indicate only a small influence of this effect. For $\mathrm{He}^{+}+\mathrm{Ne}$ at $500 \mathrm{keV} / \mathrm{u}$ we find a reduction of the theoretical total cross section by less than $3 \%$. Similar reductions were found for the other target atoms. This effect turns out to be important only for low energies.

The coupled-channel calculations presented here account for almost the full loss cross section leaving no room for a significant contribution due to correlated transitions. This is apparently in contradiction to recent results from Richard et al. [21], where it is indicated that the electron-electron and screened-Coulomb contributions for the $1 s-2 p$ excitation of $\mathrm{O}^{5+}$ by noble-gas targets $(\mathrm{Ne}, \mathrm{Ar}, \mathrm{Kr}$, and $\mathrm{Xe})$ are of the same order of magnitude. The work of Sulik et al. [22] points to a significant contribution of the electron-electron contribution for projectile excitation due to collisions with heavy targets. Nevertheless, these measurements were performed with highly charged ions for projectile velocities considerably higher than the ones studied here and only concern the excitation process. Since only small energy transfers are involved in the projectile excitation, larger impact parameters become more important in comparison with the case of projectile-electron loss. Hence, the electron-electron contribution is enhanced in the case of projectile excitation.

Correlated and uncorrelated processes are treated differently in this work. While the probability for uncorrelated transitions is calculated using the coupled-channel method, the electron-electron contributions are taken from an approximate first-order theory. It is pointed out that Eq. (14) accounts for some effects beyond first-order perturbation theory by combining correlated and uncorrelated transitions in an incoherent way. Then, the differences between the measured electron-loss cross section and the theoretical one given by Eq. (14) are most likely due either to coherence effects related to the time ordering of both processes or to nonperturbative effects in the calculation of the electronelectron contribution. For instance, the enhancement of the binding energy of the $\mathrm{He}^{+}$electron due to the strong target- 
nuclear field is taken into account for the uncorrelated transitions (through the coupled-channel method) but it is not considered in the calculations of the electron-electron contribution. The influence of a strong nuclear field on the electron-electron interaction may considerably reduce this contribution to the electron-loss process and could explain the remaining discrepancies for the $\mathrm{Ne}$ and the Ar target.

\section{CONCLUSIONS}

We have performed coupled-channel calculations of the screened-Coulomb contribution to the projectile-electron loss cross section for ${ }^{4} \mathrm{He}^{+}$on $\mathrm{He}, \mathrm{Ne}, \mathrm{Ar}$, and $\mathrm{Kr}$ in the energy range from 250 to $1000 \mathrm{keV} / \mathrm{u}$. Total cross sections are derived from the (uncorrelated) screened-Coulomb contribution and the (correlated) electron-electron contribution, calculated within a closure approximation to first-order perturbation theory.

As also found in previous works, the plane-wave-Born approximation for the screened-Coulomb contribution yields reliable electron-loss cross sections for the lightest targets (He). For other targets, the PWBA strongly overestimates the present calculations $(\mathrm{AO})$ even at high energies $(E=1000$ $\mathrm{keV} / \mathrm{u}, v=6.3$ ).

For the He target, a very good agreement is found between the calculated total and experimental electron-loss cross sections in the whole energy range. At low energies the use of the coupled-channel method slightly improves the PWBA results for the loss cross section due to the screenedtarget-nucleus projectile-electron interaction. For heavy targets we have identified an intrinsic problem in the present coupled-channel method (AO), namely, an unrealistic electron capture from $\mathrm{He}^{+}$into an occupied bound state of $\mathrm{Kr}$. This is related to the use of the independent particle model and leads to an overestimation of the electron-loss cross section at low energies for this target.

For the other targets (Ne and $\mathrm{Ar}$ ) we find good agreement between the experimental data and the coupled-channel results for uncorrelated transitions. Consequently, our results for the total electron-loss cross sections, including correlated processes, exceed the experimental data by $15 \%$ to $30 \%$. A possible influence of simultaneous electron capture and loss on the experimental results could be excluded. Thus, there are uncertainties in the theoretical treatment that increase with increasing target-nuclear charge. It is suggested that the electron-electron contribution to the electron-loss cross section for heavy targets is overestimated by the procedure of Montenegro and Meyerhof. Nonperturbative effects in the electron-electron contribution are probably responsible for the main part of the observed deviations between the present calculation and the experimental data for the heavier targets.

Finally, the present work has shown that electron-loss processes may be reasonably well described even for heavy targets if nonperturbative effects are taken into account. The experimental evidence for a target-nuclear charge-dependent saturation of electron-loss cross sections could be confirmed with our model. The remaining discrepancies, however, call for more refined theoretical models, especially for correlated transitions.

\section{ACKNOWLEDGMENTS}

The numerical work was performed on the Cray YMP-2E at the Supercomputing Center of Universidade Federal do Rio Grande do Sul (CESUP). This work was partially supported by the Brazilian agencies Conselho Nacional de Desenvolvimento Científico e Tecnológico $(\mathrm{CNPq})$ and Financiadora de Estudos e Projetos (FINEP).
[1] J.H. McGuire, N. Stolterfoht, and P.R. Simony, Phys. Rev. A 24, 97 (1981).

[2] E.C. Montenegro, W.E. Meyerhof, and J.H. McGuire, Adv. At. Mol. Opt. Phys. 34, 249 (1994).

[3] N. Stolterfoht, Nucl. Instrum. Methods B 53, 477 (1991).

[4] D.R. Bates and G.W. Griffing, Proc. Phys. Soc. A 68, 90 (1955).

[5] E.C. Montenegro, W.E. Meyerhof, J.H. McGuire, and C.L. Cocke, in The Physics of Electronic and Atomic CollisionsXIX International Conference, edited by L.J. Dub, J.B.A. Mitchell, J.W. McConkey, and C.E. Brion, AIP Conf. Proc. No. 360 (AIP Press, New York, 1995).

[6] M.M. Sant'Anna, W.S. Melo, A.C.F. Santos, G.M. Sigaud, and E.C. Montenegro, Nucl. Instrum. Methods B 99, 46 (1995).

[7] G. Schiwietz, Phys. Rev. A 42, 296 (1990).

[8] P.L. Grande and G. Schiwietz, Phys. Rev. A 44, 2984 (1991); G. Schiwietz and P.L. Grande, Nucl. Instrum. Methods B 69, 10 (1992); P.L. Grande and G. Schiwietz, Phys. Rev. A 47, 1119 (1993).

[9] J. Bang and J.M. Hansteen, Kgl. Dan. Vidensk. Selsk. Mat. Fys. Medd. 31, 13 (1959); L. Wilets and S. J. Wallace, Phys.
Rev. 169, 84 (1968); M.R. Flannery and K.J. MacCann, Phys. Rev. A 8, 2915 (1973).

[10] E.C. Montenegro and W.E. Meyerhof, Phys. Rev. A 43, 2289 (1991).

[11] F. Herman and S. Skillmann, in Atomic Structure Calculations, edited by F. Hermann and S. Skillmann (Prentice-Hall, Englewood Cliffs, NJ, 1963).

[12] A. Messiah, Quantum Mechanics (North-Holland, New York, 1963), Vol. 2.

[13] Handbook of Mathematical Functions, edited by M. Abramowitz and I. Stegun, (Dover, New York, 1970).

[14] G. Baur, M. Pauli, and D. Trautmann, Nucl. Phys. A 211, 333 (1974).

[15] W. Fritsch and C.D. Lin, J. Phys. B 15, 1255 (1982).

[16] P. R. Bates and G. Griffing, Proc. Phys. Soc. A 66, 1961 (1953).

[17] W.S. Melo, M.M. Sant'Anna, A.C.F. Santos, G.M. Sigaud, and E.C. Montenegro (unpublished).

[18] D.P. Dewangan and J. Eichler, J. Phys. B 19, 2939 (1986); Dz. Belkic, R. Gayet, J. Hanssen, and A. Salin, J. Phys. B 19, 2945 (1986).

[19] M.R.C. McDowell and J.P. Coleman, Introduction to the 
Theory of Ion-Atom Collisions (North-Holland, Amsterdam, 1970), Chap. 8.

[20] R.D. DuBois, Phys. Rev. A 39, 4440 (1989).

[21] P. Richard, G. Toth, E.C. Montenegro, T.J.M. Zouros, S. Hagmann, S.R. Grabbe, and C.P. Bhalla, in The Physics of Elec- tronic and Atomic Collisions-XIX International Conference (Ref. [5]).

[22] B. Sulik, S. Ricz, I. Kadar, G. Xiao, G. Schiwietz, K. Sommer, P.L. Grande, R. Koehrbrueck, M. Grether, and N. Stolterfoht, Phys. Rev. A 52, 387 (1995). 